\title{
Reaction Behavior of Facing Pair between Hematite and Graphite: A Coupling Phenomenon of Reduction and Gasification
}

\author{
Yoshiaki KASHIWAYA, Motomichi KANBE") and Kuniyoshi ISHII \\ Graduate School of Engineering, Hokkaido University. \\ Hokkaido University. E-mail: Yoshiaki@eng.hokudai.ac.jp \\ 1) Student of Master course, Graduate School of Engineering,
}

(Received on March 13, 2001; accepted in final form on April 19, 2001)

\begin{abstract}
Recently, the reaction of composite pellet consisting of the iron ore and carbonaceous materials such as coal, char and coke was investigated. The fast reaction rate and relatively low starting temperature were reported by many researchers. In such a reaction condition, the mechanism of reaction will be changed during reaction from the situation that the iron ore and carbon are directly contacting and to the separate situation.

The simultaneous reaction between reduction and gasification of carbon was examined using hematitegraphite facing pair, which is to clarify the effect of reaction occurring in the separated place. From the definition of coupling phenomenon, the discussion about the 'produced energy' related to the production of entropy was carried out in view point of two aspects that were (a) the gas composition change and (b) the evolution of reaction heat.

The starting temperature of gasification decreased from $900^{\circ} \mathrm{C}$ to $600^{\circ} \mathrm{C}$ in the facing pair under $\mathrm{CO}_{2}$ atmosphere and to $250^{\circ} \mathrm{C}$ under $\mathrm{CO}$ atmosphere. The effect of reaction heat of reduction was measured and discussed through new mechanism of reduction.
\end{abstract}

KEY WORDS: coupling reaction; coupling phenomenon; iron ore reduction; gasification; mechanism of reduction.

\section{Introduction}

The existence of iron would be a matter of course such as air and water at present industrial society. The iron and its products are important and fundamental materials to sustain the base of society.

Now, most of iron and steel are made by the blast furnace and converter process. The reason that the blast furnace method is keeping the position as a major process for ironmaking is the reactor having highest efficiency on the reactions and heat exchange in spite of the biggest reactor comparing with the other chemical one.

However, even in such a blast furnace process, there might be some remained possibility to increase the efficiency from the viewpoint of thermodynamics. If the rate of reactions could increase and the starting temperature of gasification reaction could be lowered, further increase of the efficiency in the blast furnace process could be expected. For example, the thermal and the chemical reserve zones in the blast furnace would be a kind of inactive zone and make the reactor biggest. The both reserve zones are generated spontaneously from the thermodynamics and reaction mechanism between the reduction of iron ore and coke gasification.

Recently, composite pellet of iron ore and carbonaceous material (e.g. coal, char and coke) has been developed and used for new process such as FASTMET. The reaction behavior of the composite pellet was energetically investigated, in which the higher rate of reaction both of reduction and gasification and the low starting temperature of gasification were reported.

One of reason of this phenomenon might be resulted from a kind of recycle of product gas which could be explained by reactions (1) and (2).

$$
\begin{array}{r}
\mathrm{FeO}_{x}+\mathrm{CO}=\mathrm{FeO}_{x-1}+\mathrm{CO}_{2} \\
\mathrm{C}+\mathrm{CO}_{2}=2 \mathrm{CO} . \ldots \ldots \ldots
\end{array}
$$

While the overall reaction can be explained by Reaction (3) which is so-called direct reduction.

$$
\mathrm{FeO}_{x}+\mathrm{C}=\mathrm{FeO}_{x-1}+\mathrm{CO} \text {. }
$$

The initiation of the reaction should be the reaction (3) in the case of composite pellet under inert atmosphere (Fig. 1(a)). However, the mechanism of subsequent reaction

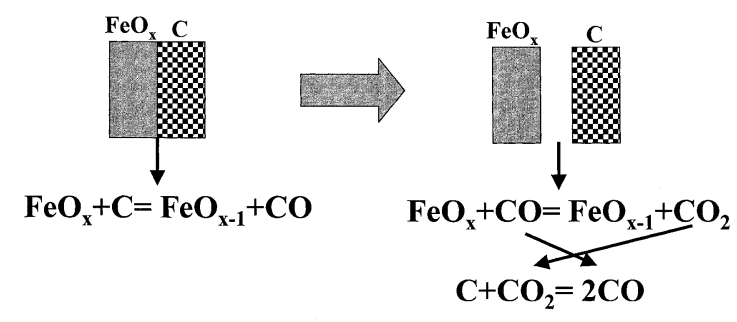
(a) Contact
(b) Separate

Fig. 1. Illustration of the change of reaction mechanism in the composite pellet. 
might be different and changed by the location of carbon and iron ore. Once the position between carbon and iron ore was separated, the reaction (3) never occur and reactions (1) and (2) should be dominant (Fig. 1(b)). However, it is a common situation of the reduction and gasification and very difficult to explain the reason why the rate of reaction is accelerated from the only recycling of product gases. Only one possibility might be deduced by the location between the iron ore and carbon where the distance between iron ore and carbon is short and reaction interfaces are facing each other.

In this study, the reaction behavior in the situation that the iron ore and carbon are separated is investigated. The rates of reduction reaction and gasification reaction were evaluated separately by the gas analysis using QMS (Quadrupole Mass Spectrometer). The effect of a coupling phenomenon between the reduction and gasification on the overall rate was estimated. Furthermore, the temperature variations of the reaction surfaces were measured and a reaction mechanism was proposed.

\section{Definition of Thermodynamic Coupling and Cou- pling Reaction ${ }^{1,2)}$}

According to the Second Low of Thermodynamics, the entropy change of the closed system in a reversible process can be expressed as follows ${ }^{1)}$ :

$$
d S=d Q / T \quad(\text { reversible process })
$$

For the irreversible process, the entropy change of the system is larger than the $d Q / T$ :

$$
d S \geqq d Q / T \quad \text { (irreversible process). }
$$

Clausius has introduced $d Q^{\prime}$ called the 'uncompensated heat' which was generated in the irreversible process. Then, the total entropy change in the system can be expressed as Eq. (6).

$$
d S=d Q / T+d Q^{\prime} / T
$$

( $d Q^{\prime}=0$ : reversible process, $d Q^{\prime}>0$ : irreversible process)

Hence, we can classify the energy (heat and work) in the irreversible process. One is the energy transfer from the external system into the system, which is expressed as $d Q e$, the other is the energy produced in the system according to the irreversible process, which is expressed as $d Q i$. Then the change of entropy can be expressed as:

$$
d S=d S e+d S i=d Q e / T+d Q i / T .
$$

where $d S e$ means the 'transport of entropy' from external system into the system and $d S i$ means the 'production of entropy' in the system.

De Donder ${ }^{2)}$ has derived the inequality (Eq. (8)) expressed the relationship between the 'produced energy', $d Q i$ and the affinity of reaction ' $x$ ', $A_{x}$.

$$
d Q i=A_{x} d \xi \geqq 0
$$

As mentioned above, the $d Q i$ was used to call as 'uncompensated heat', however, it was relatively difficult expression. In present paper, the simple expression 'produced energy' was used, that the correct meaning would be 'pro- duced energy related to the production of entropy'. The ' $\xi$ ' means the 'extent of reaction' ${ }^{1,3,4)}$ As the $d \xi / d t$ is related to the rate of reaction $\left(v_{x}\right.$ : rate of reaction ' $x$ ', $\left.\mathrm{mol} / \mathrm{s}\right)$,

$$
d \xi / d t=v_{x}
$$

the rate of produced energy can be expressed as Eq. (10).

$$
d Q i / d t=A_{x} d \xi / d t=A_{x} v_{x} \geqq 0 .
$$

In case of simultaneous reaction, the rate of produced energy can be rewritten as follows;

$$
d Q i / d t=\sum A_{n} v_{n} \geqq 0 \quad(n=1,2,3 \ldots)
$$

The important point of Eq. (11) is that the summation of $A_{n} v_{n}$ should be positive regardless of $A_{1} v_{1}<0$ or $A_{2} v_{2}<0$.

If the first reaction is $A_{1} v_{1}<0$ and the second reaction is $A_{2} v_{2}>0$, the first reaction ' 1 ' is called as 'Coupled reaction' and second reaction is 'Coupling reaction'. The generic expression of this phenomenon is 'Thermodynamic coupling'. In general, the word 'coupling reaction' was used as the thermodynamic coupling, because the word 'coupling reaction' would be a convenient and comprehensive word. In this paper, we will use the words 'coupling phenomenon' and 'thermodynamic coupling' for the generic expression for classifying from both of the 'coupled reaction' and 'coupling reaction'.

On the other hand, the affinity of a reaction (12) can be related to the free energy $(d G)$ and Chemical potential $(\mu)$ as shown by Eq. (13). ${ }^{2-4)}$

$$
v_{1} R_{1}+v_{2} R_{2}=v_{3} P_{3}+v_{4} P_{4} .
$$

Where $v_{\mathrm{i}}$ is the stoichiometric coefficient of $i$ component. $R_{1}$ and $R_{2}$ are the reactants and $P_{1}$ and $P_{2}$ are the products.

$$
A=-(d G / d \xi)_{\mathrm{P}}=-\sum v_{\mathrm{i}} \mu_{\mathrm{i}}
$$

Where $\mu_{i}$ is the chemical potential of ' $i$ ' component. The difference of free energy $\Delta G$ (the difference between the products and the reactants) in a nonequilibrium state can be expressed by Eq. (14).

$$
\begin{aligned}
\Delta G=\sum v_{i} \mu_{i} & =v_{3} \mu_{4}+v_{4} \mu_{4}-v_{1} \mu_{1}-v_{2} \mu_{2} \\
& =\Delta G^{\circ}+R T \ln K p \ldots \ldots \ldots \ldots \ldots \ldots \ldots \ldots \ldots
\end{aligned}
$$

Where $\Delta G^{\circ}$ is standard free energy of a reaction and $K p$ is the gas constant $\left(K p=\left(a_{\mathrm{P} 3}^{\nu 3} \cdot a_{\mathrm{P} 4}^{\nu 4}\right) /\left(a_{\mathrm{R} 1}^{\nu 1} \cdot a_{\mathrm{R} 2}^{\nu 2}\right)\right.$, which is equal to equilibrium constant when the reaction reaches to the equilibrium state).

Hence, the relationship between the affinity ' $A$ ' and the change of free energy ' $\Delta G$ ' is as follow:

$$
A=-\Delta G=-\left(\Delta G^{\circ}+R T \ln K p\right)
$$

In case of equilibrium state, the affinity is equal to zero $(A=$ $-\Delta G=0)$ and the common expression $\left(\Delta G^{\circ}=-R T \ln K p\right)$ can be obtained.

In this study, the coupling phenomenon between the reduction and the gasification will be investigated. The Eq. (11) can be rewritten as follows;

$$
A_{\mathrm{R}} v_{\mathrm{R}}+A_{\mathrm{G}} v_{\mathrm{G}}>0
$$


Where $A_{\mathrm{R}}$ and $A_{\mathrm{G}}(\mathrm{J} / \mathrm{mol})$, which are defined by Eqs. (17) and (18), are chemical affinity of reduction and gasification, respectively. $v_{\mathrm{R}}$ and $v_{\mathrm{G}}(\mathrm{mol} / \mathrm{s})$ are the rate of reduction and gasification, respectively.

$$
\begin{aligned}
& A_{\mathrm{R}}=-\Delta G_{\mathrm{R}}=-\left(\Delta G_{\mathrm{R}}^{\circ}+R T \ln \frac{P_{\mathrm{CO}_{2}}}{P_{\mathrm{CO}}}\right) \ldots \ldots \ldots . . . \\
& A_{\mathrm{G}}=-\Delta G_{\mathrm{G}}=-\left(\Delta G_{\mathrm{G}}^{\circ}+R T \ln \frac{P_{\mathrm{CO}}^{2}}{P_{\mathrm{CO}_{2}}}\right) \ldots \ldots \ldots . .
\end{aligned}
$$

Where $\Delta G_{\mathrm{R}}^{\circ}$ and $\Delta G_{\mathrm{G}}^{\circ}$ are the standard free energy of reduction and gasification, respectively.

The $A_{\mathrm{R}}$ and $A_{\mathrm{G}}\left(\Delta G_{\mathrm{R}}^{\circ}\right.$ and $\left.\Delta G_{\mathrm{G}}^{\circ}\right)$ were calculated using thermodynamic data. ${ }^{5)}$ The reduction reaction will change in the course of experiment and the calculation of $A_{\mathrm{R}}$ (actually $\Delta G_{\mathrm{R}}^{\circ}$ ) was changed with the degree of reduction (TRD) as follows:

$$
\begin{gathered}
3 \mathrm{Fe}_{2} \mathrm{O}_{3}+\mathrm{CO}=2 \mathrm{Fe}_{3} \mathrm{O}_{4}+\mathrm{CO}_{2}(\mathrm{TRD} \leqq 11 \%) \ldots \ldots \ldots . . \\
\mathrm{Fe}_{3} \mathrm{O}_{4}+\mathrm{CO}=3 \mathrm{FeO}+\mathrm{CO}_{2}(11 \%<\mathrm{TRD} \leqq 33.3 \%) \ldots \ldots \\
\mathrm{FeO}+\mathrm{CO}=\mathrm{Fe}+\mathrm{CO}_{2}(\mathrm{TRD}>33.3 \%) \ldots \ldots \ldots \ldots . . .
\end{gathered}
$$

Strictly, the reduction mechanism should be examined and the above reaction might be changed according to the mechanism (e.g. Shrinking Core Model such as Three Interface Model or Homogeneous Model). However, the purpose of present study is to clarify the phenomena between the reduction and the gasification in a separated condition (The quantitative discussion will be carried out in future work). Further, the profile of the rate of reduction roughly changed with the TRD at $11 \%$ and $33 \%$ (e.g. Fig. 7 , the detail will be mentioned in the later section).

The partial pressure of $\mathrm{CO}$ and $\mathrm{CO}_{2}$ were obtained from the flow rates of gases in the experiment $\left(P_{\mathrm{CO}}=V_{\mathrm{CO}} / V_{\mathrm{T}}\right.$, $P_{\mathrm{CO}_{2}}=V_{\mathrm{CO}_{2}} / V_{\mathrm{T}}, V_{\mathrm{CO}}, V_{\mathrm{CO}_{2}}(\mathrm{Ncc} / \mathrm{min})$ : flow rates of gases, $V_{\mathrm{T}}$ ( Ncc/min): total flow rate). In the later discussion, the affinity of a reaction was considered using inlet gas and outlet gas, so that $A_{\text {in }}\left(A_{\text {out }}\right)$ was calculated using $P_{\mathrm{CO}}$ and $P_{\mathrm{CO}_{2}}$ from inlet (outlet) gas composition.

As mentioned above, the inlet gas does not always flow into the space between the samples. Some gas would react with the rear and side surface of sample, which might dilute the coupling phenomenon. Although some extent of coupling phenomenon could be detected in this experiment as a result, the actual amount of the phenomenon should be larger than the present analysis.

From above definition, the discussion of coupling phenomenon will be focused on the following points;

(1) The gas composition change caused by reaction, which related to the entropy term.

(2) The change of reaction heat, which related to the enthalpy term.

\section{Experimental}

Figure 2 shows the schematics of the reaction furnace used for present study. The furnace was a Gold Image Furnace which heated the sample by infrared lump. Reaction tube was made of quartz having water cooling cap

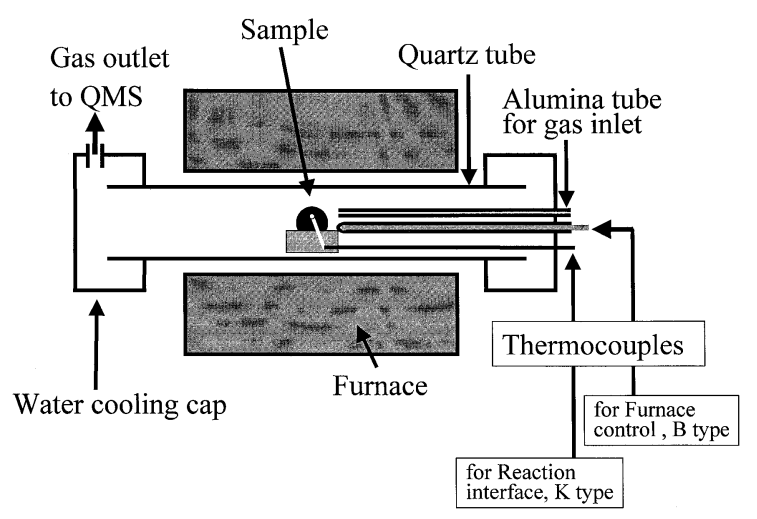

Fig. 2. Schematics of reaction furnace.

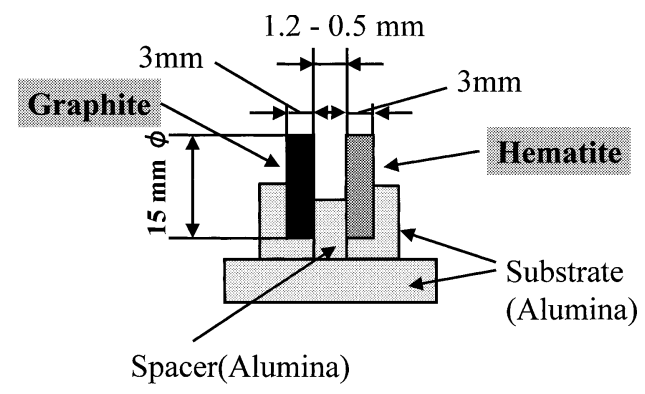

Fig. 3. Alignment of graphite and hematite sample.

at the both ends for the gas sealing by O-rings.

The reaction gas was introduced by alumina tube $(6 \mathrm{~mm} \phi$ O.D. $\times 4 \mathrm{~mm} \phi$ I.D.) and the outlet of the tube was closed to the sample edge by $5 \mathrm{~mm}$. The exit gas was analyzed by QMS (Quadrupole Mass Spectrometer). ${ }^{6-9)}$ The temperature of furnace was controlled by B-type thermocouple (Pt-6\%Rh, Pt-30\%Rh), which was $0.5 \mathrm{~mm}$ in diameter and set in the furnace using a alumina protection tube $(6 \mathrm{~mm} \phi$ O.D.). While the temperature of reaction surfaces were measured by K-type (CA, $0.3 \mathrm{~mm} \phi)$ thermocouples without protection tube during experiment to detect the reaction heat in several experiments.

Figure 3 shows the alignment of samples (graphite and hematite) on the alumina substrate. The distance $(1.2 \mathrm{~mm}$ or $0.5 \mathrm{~mm}$ ) between two samples was adjusted by the thickness of alumina spacer. In this paper, the discussions were mainly carried out using the results from $1.2 \mathrm{~mm}$ distance of samples, because a little gas flowed into the space of two samples in the case of $0.5 \mathrm{~mm}$. As a result, the amount of reaction in $0.5 \mathrm{~mm}$ decreased corresponding to that of $1.2 \mathrm{~mm}$. However, as the qualitative tendency was the same in the both conditions, so that most of discussion was carried out with the data in the distance of $1.2 \mathrm{~mm}$.

The heat of reaction will affect the overall reaction rate each other. Then the variations of temperatures at both the surfaces of samples were measured by K-type thermocouple $(0.3 \mathrm{~mm} \phi)$. The positions of thermocouples were shown in Fig. 4. The tip of thermocouple was fixed by alumina cement from the rear side of sample and about $50 \%$ of surface of tip was exposed to the gas beyond the reaction surface.

The hematite sample was prepared from a reagent grade hematite and compressed into disk shape $(15 \mathrm{~mm} \phi \times 5 \mathrm{~mm})$ in the mold with $200 \mathrm{kgf} / \mathrm{cm}^{2}$. Then, the disk shape hematite was sintered in the muffle furnace at $1200^{\circ} \mathrm{C}$ for 


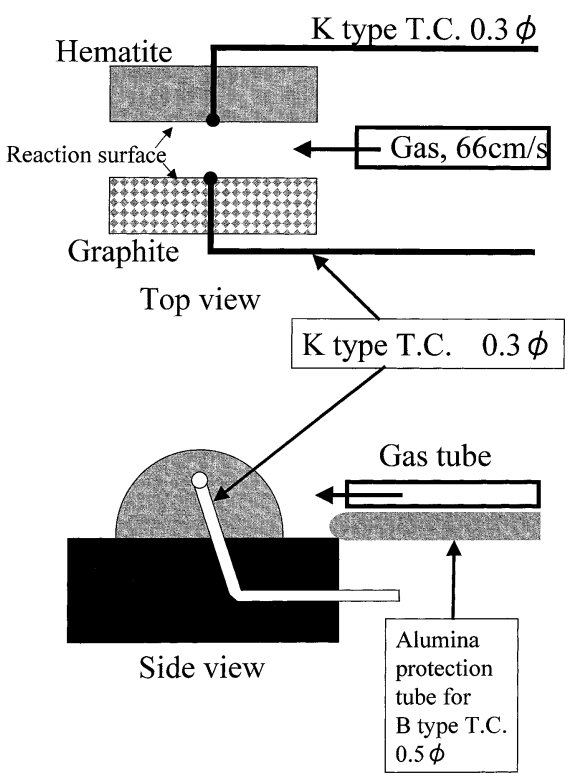

Fig. 4. Position of thermocouples for measurement of the temperature change at the reaction surface.

24 hours. The sintered sample was adjusted the thickness to $3 \mathrm{~mm}$ by grinding and the reaction surface was polished until \#1200 using SiC paper. The reaction gas was $500 \mathrm{Ncc} / \mathrm{min}(66 \mathrm{~cm} / \mathrm{s}$ at the exit of alumina gas inlet tube) in total and the compositions were $\mathrm{Ar}-30 \mathrm{vol} \% \mathrm{CO}$ which was the reaction gas for the initiation of reduction reaction and $\mathrm{Ar}-30 \mathrm{vol} \% \mathrm{CO}_{2}$ which was for that of gasification reaction. Heating up rate was $15^{\circ} \mathrm{C} / \mathrm{min}$ from ambient temperature to $1000^{\circ} \mathrm{C}$ and held at $1000^{\circ} \mathrm{C}$ for $30 \mathrm{~min}$. The variation of the gas composition was continuously monitored by QMS and the rate of reactions were calculated after each experiment. The standard gas $\left(40 \mathrm{vol} \% \mathrm{CO}, 20 \mathrm{vol} \% \mathrm{CO}_{2}\right.$ argon balance) was used for calibration of QMS output that was performed before and after experiments, because the condition of QMS (the amount of gas adsorption inside the container tube of quadrupole) was changed with time. The precise control of temperature around QMS made the high accurate measurement possible and as the sensitivity of QMS was quite high ( $\geqq 10 \mathrm{ppm}$ ), small amount of reaction can be detected, and the accuracy of measurement was $0.1 \%$ for the total flow rate. Then, in this experiment, $0.5 \mathrm{Ncc} / \mathrm{min}$ flow rate can be detected, which corresponded to $0.037 \times 10^{-5}(\mathrm{~mol} / \mathrm{s})$ as a reduction rate $(\mathrm{RDR})$.

The rate of reduction (RDR) and gasification (RCS) can be obtained by the oxygen balance and carbon balance in the reactant and product gases, respectively. RDR and RCS are equal to $v_{\mathrm{R}}$ and $v_{\mathrm{G}}(\mathrm{mol} / \mathrm{s})\left(\mathrm{RDR}=v_{\mathrm{R}}, \mathrm{RCS}=v_{\mathrm{G}}\right)$ for the calculation of $A_{\mathrm{R}} v_{\mathrm{R}}+A_{\mathrm{G}} v_{\mathrm{G}}$, respectively.

$$
\begin{aligned}
\operatorname{RDR}(\mathrm{mol} / \mathrm{s})= & \left([\mathrm{CO}]_{\text {out }}+2\left[\mathrm{CO}_{2}\right]_{\text {out }}-[\mathrm{CO}]_{\text {in }}\right. \\
& \left.-2\left[\mathrm{CO}_{2}\right]_{\text {in }}\right) / 22412 / 60 \ldots \ldots . . \\
\operatorname{RCS}(\mathrm{mol} / \mathrm{s})= & \left([\mathrm{CO}]_{\text {out }}+\left[\mathrm{CO}_{2}\right]_{\text {out }}-[\mathrm{CO}]_{\text {in }}\right. \\
& \left.-\left[\mathrm{CO}_{2}\right]_{\text {in }}\right) / 22412 / 60 \ldots \ldots \ldots . . .
\end{aligned}
$$

Where [CO] and $\left[\mathrm{CO}_{2}\right]$ mean the mass flowrate of respective gases in Ncc/min and subscripts 'in' and 'out' mean the inlet and the outlet. The degree of reduction (TRD) and gasification (TCS) were obtained by the integration of RDR

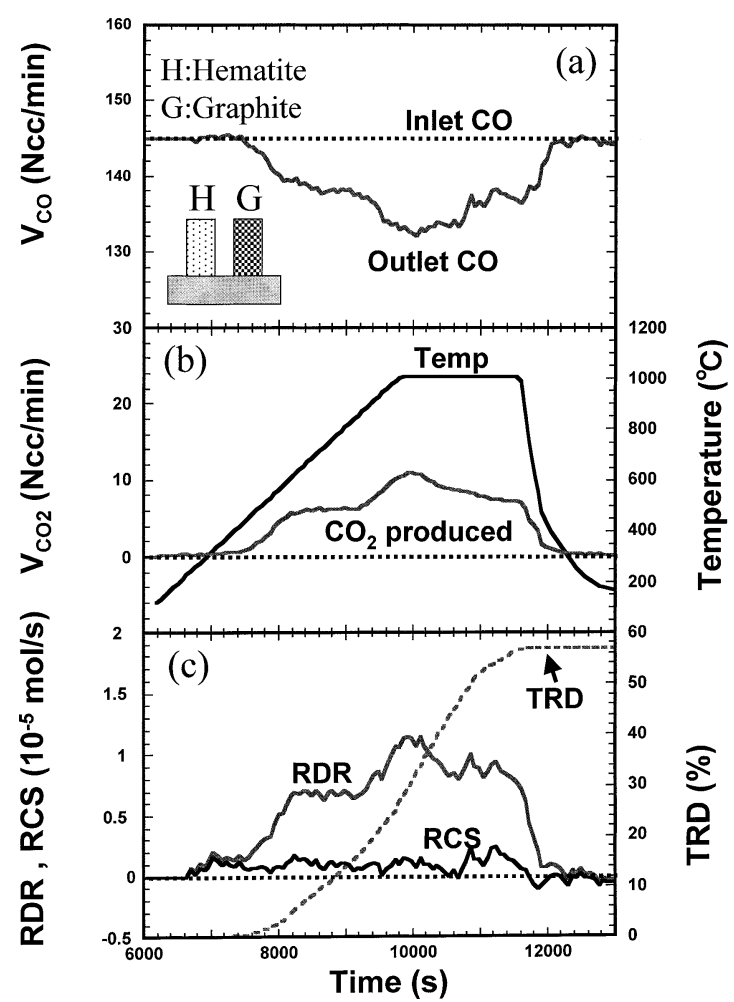

Fig. 5. Variation of $\mathrm{CO}$ and $\mathrm{CO}_{2}$ flowrates and reaction rates (RDR, RCS). (facing pair, $1.2 \mathrm{~mm}, \mathrm{Ar}-30 \mathrm{vol} \% \mathrm{CO}$ )

and RCS with time, respectively.

$$
\begin{aligned}
& \operatorname{TRD}(\%)=\left(100 /[\mathrm{O}]_{0}\right) \sum \mathrm{RDR} \cdot \Delta t \\
& \operatorname{TCS}(\%)=\left(100 /[\mathrm{C}]_{0}\right) \sum \mathrm{RCS} \cdot \Delta t
\end{aligned}
$$

Where $[\mathrm{O}]_{0}$ and $[\mathrm{C}]_{0}$ represent the reducible oxygen and total fixed carbon before experiment, respectively.

\section{Results and Discussions}

\subsection{Assessment of Coupling Phenomenon from Gas Composition Change}

Figure 5 shows the variation of flowrate of gases (CO and $\mathrm{CO}_{2}$ ) during experiment. RDR and RCS were calculated using Eqs. (22) and (23). The samples were the facing pair of graphite and hematite and the distance was $1.2 \mathrm{~mm}$. The composition of reaction gas was $\mathrm{Ar}-30 \mathrm{vol} \% \mathrm{CO}$ that corresponded to the gas composition for the initiation of reduction (only reduction will occur from the composition of gas). The $\mathrm{CO}$ gas (Fig. 5(a)) decreased from the inlet gas level because of reduction reaction and $\mathrm{CO}_{2}$ (Fig. 5(b)) evolved during experiment. The rate of reduction (RDR) occurred from about $250^{\circ} \mathrm{C}$ (Fig. 5(c), Table 1). Although the amount of gasification was small, the rate of gasification (RCS) was also observed from the same temperature. Since the $\mathrm{CO}_{2}$ was not contained in the inlet gas, it is no doubt that the gasification reaction was resulted from the $\mathrm{CO}_{2}$ produced in the reduction reaction which occurred in the opposite and distant surface. Furthermore, the temperature $250^{\circ} \mathrm{C}$ was very low and it would be impossible to proceed the gasification thermodynamically. The amount of TRD obtained by QMS was almost equal to the weight loss 


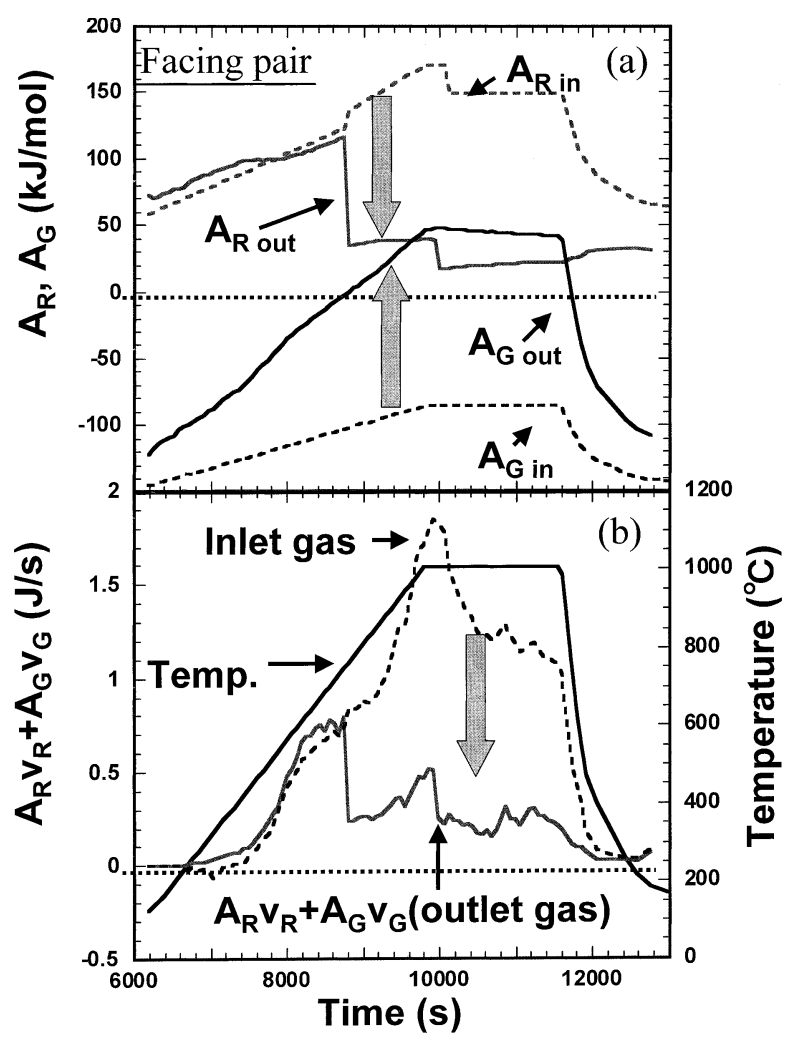

Fig. 6. Variation of $A_{\mathrm{R}}, A_{\mathrm{G}}$ and $A_{\mathrm{R}} v_{\mathrm{R}}+A_{\mathrm{G}} v_{\mathrm{G}}$ (facing pair, $1.2 \mathrm{~mm}$, $\mathrm{Ar}-30 \mathrm{vol} \% \mathrm{CO})$

of hematite after experiment.

Using the definition of thermodynamic coupling, the affinities of each reaction, $A_{\mathrm{R}}$ and $A_{\mathrm{G}}$ were calculated and the produced energy $\left(A_{\mathrm{R}} v_{\mathrm{R}}+A_{\mathrm{G}} v_{\mathrm{G}}\right)$ was evaluated. Those results are shown in Fig. 6. In present study, the affinities of each reaction were calculated using the inlet and outlet gas compositions ( $A_{\text {in }}$ and $\left.A_{\text {out }}\right)$. The broken line in Fig. 6 means the affinities of inlet gas $\left(A_{\text {in }}\right)$ for each reactions $\left(P_{\mathrm{CO}_{2}}\right.$ was assumed $\left.10^{-7}\right)$ and the solid line means that of outlet gas $\left(A_{\text {out }}\right)$. As the inlet gas did not contain $\mathrm{CO}_{2}$ gas, the affinity of gasification $A_{\mathrm{G} \text {,in }}$ was negative and large absolute value ( $-150--100 \mathrm{~kJ} / \mathrm{mol}$, Fig. 6(a)), which meant the gasification reaction never occurred in view point of inlet gas. However, the affinity $A_{\mathrm{G} \text {,out }}$ in the outlet gas crossed the zero at $700^{\circ} \mathrm{C}$, which meant that the possibility to occur the gasification reaction generated from $700^{\circ} \mathrm{C}$ by the $\mathrm{CO}_{2}$ evolution from reduction. On the other hand, the affinity of reduction $A_{\mathrm{R}}$ decreased in the outlet gas with increase of temperature because the rate of reduction increased and produced gas $\mathrm{CO}_{2}$ increased. However, $A_{\mathrm{R}}$ was never beyond zero and always positive (the calculation of $A_{\mathrm{R}}$ was changed by the reduction degree TRD according to hematite $(<11 \%)$, magnetite $(<33 \%)$ and wustite $(>33 \%)$ reductions, Eqs. (19), (20) and (21). Since the value of $A_{\mathrm{R}} v_{\mathrm{R}}+A_{\mathrm{G}} v_{\mathrm{G}}$ was always positive, the coupling phenomenon could be occurred from room temperature regarding to the inlet gas and until $700^{\circ} \mathrm{C}$ in view point of the outlet gas. It could be concluded that the rate of gasification (RCS) observed from $250^{\circ} \mathrm{C}$ generated from the consequence of coupling phenomenon between reduction and gasification. In this case, the reduction reaction is the coupling reaction and the gasification is the coupled reaction.

The single hematite reduction was carried out using alu-

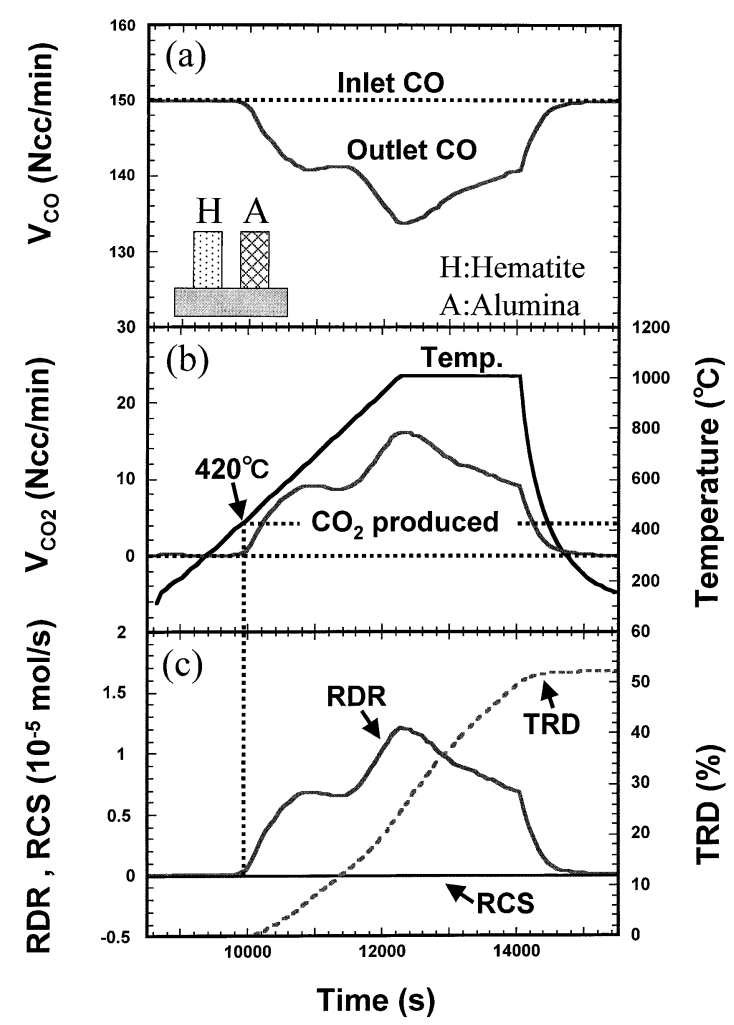

Fig. 7. Variation of $\mathrm{CO}$ and $\mathrm{CO}_{2}$ flowrates and reaction rates (RDR, RCS). (single hematite, Ar-30vol\%CO)

Table 1. Comparison of starting temperature among different experiments.

\begin{tabular}{|c|c|c|c|}
\hline \multirow{2}{*}{ Gas Composition } & \multirow{2}{*}{ Samples } & \multicolumn{2}{|c|}{ Starting temperature } \\
\hline & & RDR & RCS \\
\hline \multirow{2}{*}{$\mathrm{Ar}-30 \mathrm{vol} \% \mathrm{CO}$} & $\begin{array}{l}\text { Hematite-Graphite } \\
\text { Facing pair }\end{array}$ & $250-300^{\circ} \mathrm{C}$ & $250-300^{\circ} \mathrm{C}$ \\
\hline & Hematite single & $420^{\circ} \mathrm{C}$ & - \\
\hline \multirow{2}{*}{$\mathrm{Ar}-30 \mathrm{vol} \% \mathrm{CO}_{2}$} & $\begin{array}{l}\text { Hematite-Graphite } \\
\text { Facing pair }\end{array}$ & $600^{\circ} \mathrm{C}$ & $600^{\circ} \mathrm{C}$ \\
\hline & Graphite single & - & $900^{\circ} \mathrm{C}$ \\
\hline
\end{tabular}

mina disk instead of graphite for the comparison of facing pair. Figure 7 shows the variation of gas composition and reduction rate (RDR) during experiment. In this case, it was natural that the gasification was zero because of no carbonaceous material in the system $(\mathrm{RCS}=0)$. The gas composition changes were observed from $420^{\circ} \mathrm{C}$ and the reduction (RDR) occurred from the same temperature (Table 1). As the RDR was observed from $300^{\circ} \mathrm{C}$ in the case of facing pair (Fig. 5), it could emphasize that the reduction was promoted by coupling phenomenon in low temperature range. Figure 8 shows the comparison of RDR and RCS between the facing pair and the single hematite. It should be noted that the apparent reduction curves (RDR) were almost the same between the facing pair and the single hematite and each steps in the RDR curve of single hematite will correspond to the respective reduction steps, hematite, magnetite and wustite, roughly. Only small step from 300 to $420^{\circ} \mathrm{C}$ in the facing pair was observed and promoted by the coupling phenomenon because the diffusion of $\mathrm{CO}$ and $\mathrm{CO}_{2}$ to/from the reaction interface through the product layer would be 


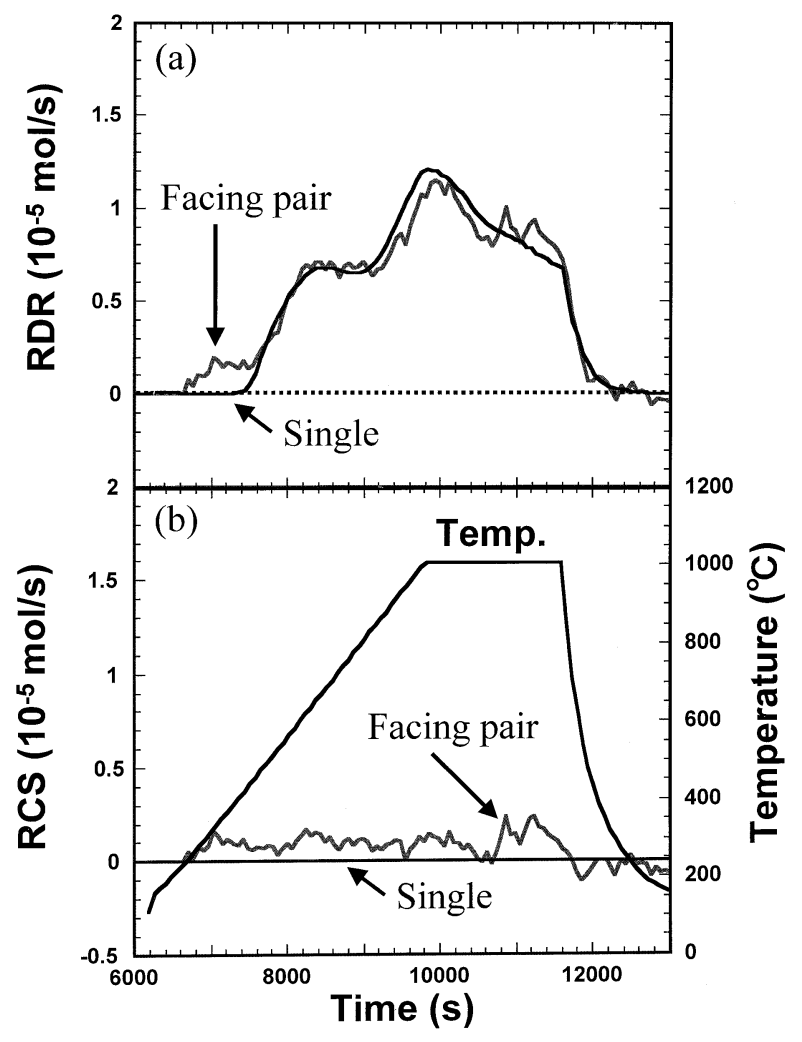

Fig. 8. Comparison of reaction rates (RDR, RCS) between the facing pair and the single hematite in $\mathrm{Ar}-30 \mathrm{vol} \% \mathrm{CO}$.

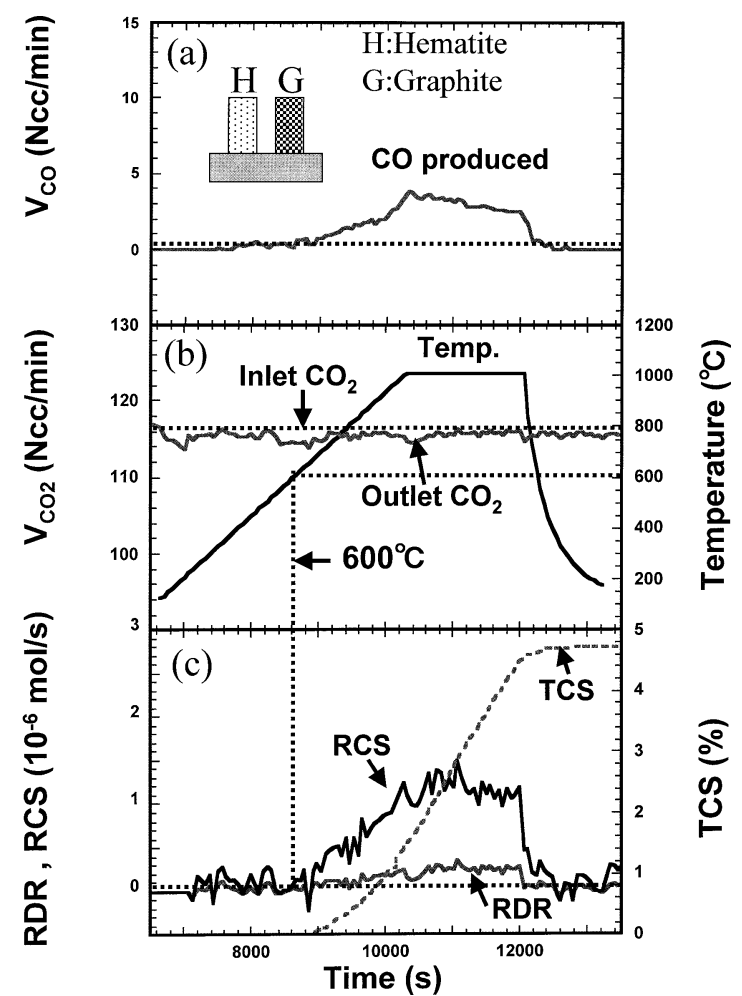

Fig. 9. Variation of $\mathrm{CO}$ and $\mathrm{CO}_{2}$ flowrates and reaction rates (RDR, RCS). (facing pair, $1.2 \mathrm{~mm}, \mathrm{Ar}-30 \mathrm{vol} \% \mathrm{CO}_{2}$ )

dominant in the course of reduction.

Figure 9 shows the results of facing pair between hematite and graphite in $\mathrm{Ar}-\mathrm{CO}_{2}$ mixture, which is the gas composition for the initiation of gasification. The gasification reaction $\mathrm{RCS}$ occurred from $600^{\circ} \mathrm{C}$ (Table 1) which

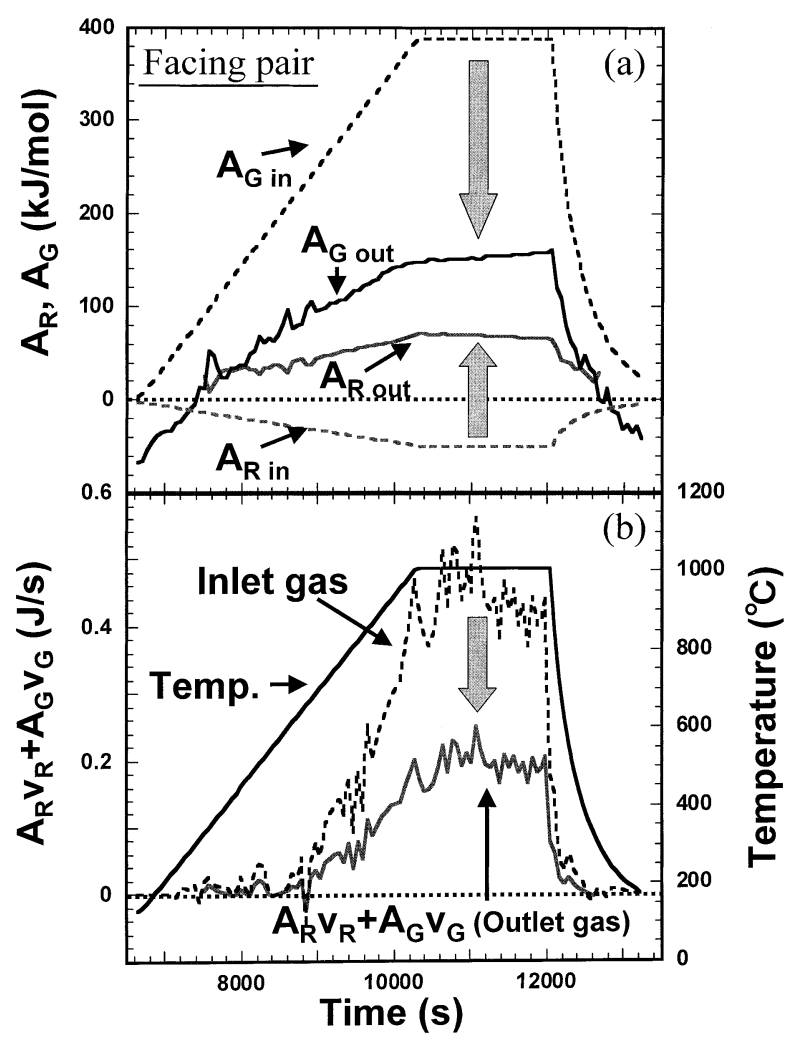

Fig. 10. Variation of $A_{\mathrm{R}}, A_{\mathrm{G}}$ and $A_{\mathrm{R}} v_{\mathrm{R}}+A_{\mathrm{G}} v_{\mathrm{G}}$ (facing pair $1.2 \mathrm{~mm}, \mathrm{Ar}-30 \mathrm{vol} \% \mathrm{CO}_{2}$ )

was quite low temperature to start the gasification of graphite. The reduction RDR also began to increase from the same temperature $\left(600^{\circ} \mathrm{C}\right)$, although it was a small amount.

The same plots as Fig. 6 to analyze the coupling phenomenon were made using the thermodynamic data from literature $^{5)}$ and each gas compositions (inlet and outlet) observed and shown in Fig. 10. The affinity of reduction in the inlet gas $A_{\mathrm{R}, \text { in }}$ was always negative, which meant the reduction reaction never occur and the one in the outlet gas $A_{\mathrm{R}, \text { out }}$ turned to positive. The affinities of gasification reaction $\left(A_{\mathrm{G}, \text { in }}, A_{\mathrm{G}, \text { out }}\right)$ were always positive regardless to the inlet and outlet gas composition, although the extent of $A_{\mathrm{G}, \text { out }}$ decreased in the outlet gas. Moreover, the rate of produced energy, $A_{\mathrm{R}} v_{\mathrm{R}}+A_{\mathrm{G}} v_{\mathrm{G}}$, was always positive and decreased in the outlet gas. In this case, the condition of coupling phenomenon was not satisfied, because both of $A_{\mathrm{R}, \text { out }}$ and $A_{\mathrm{G} \text {,out }}$ were positive regarding to the outlet gas. However, in the view point of the inlet gas, the coupling phenomenon could be present owing to the negative value of $A_{\mathrm{R} \text {,in }}$.

The single graphite experiment was carried out for the comparison of reaction rate between the facing pair and the single graphite, in which the hematite sample was changed to alumina. Figure 11 show the results of the single graphite in $\mathrm{Ar}-30 \mathrm{vol} \% \mathrm{CO}_{2}$ flowrate. It was found that the gasification reaction occurred from $900^{\circ} \mathrm{C}$, which was about $300^{\circ} \mathrm{C}$ higher than that in the facing pair (The starting temperature of gasification was $600^{\circ} \mathrm{C}$ in the facing pair, Table 1).

Figure 12 shows the comparison of reaction rate (RDR, RCS) between the facing pair and the single graphite. As mentioned above, the all introduced gas did not flow into 


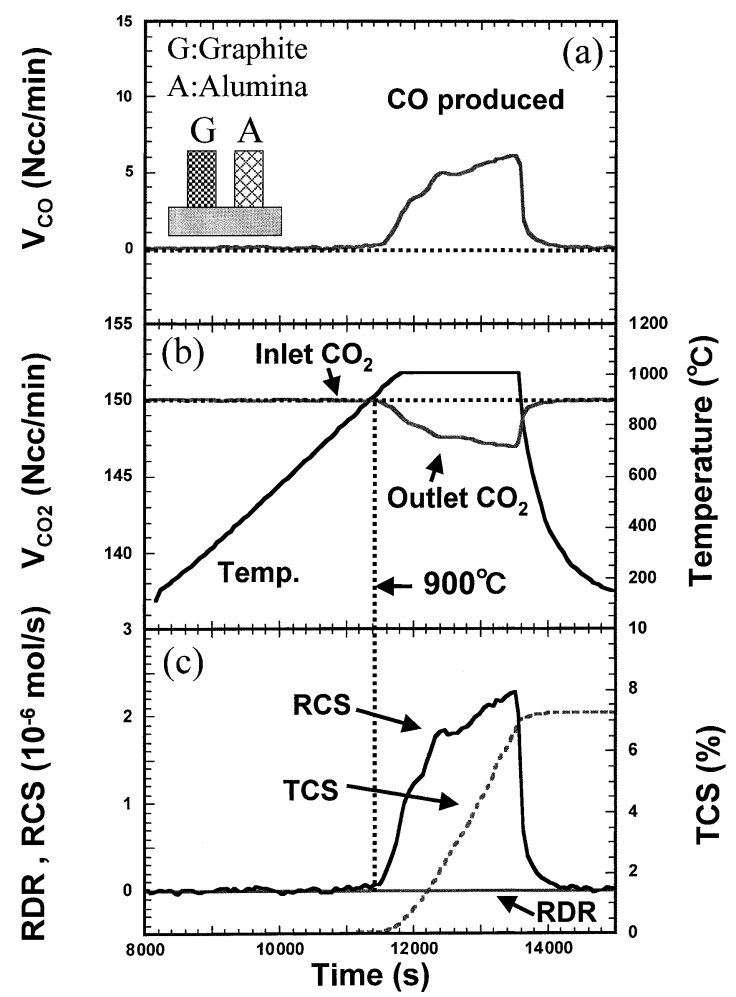

Fig. 11. Variation of $\mathrm{CO}$ and $\mathrm{CO}_{2}$ flowrates and reaction rates (RDR, RCS). (single graphite, $\mathrm{Ar}-30 \mathrm{vol} \% \mathrm{CO}_{2}$ )

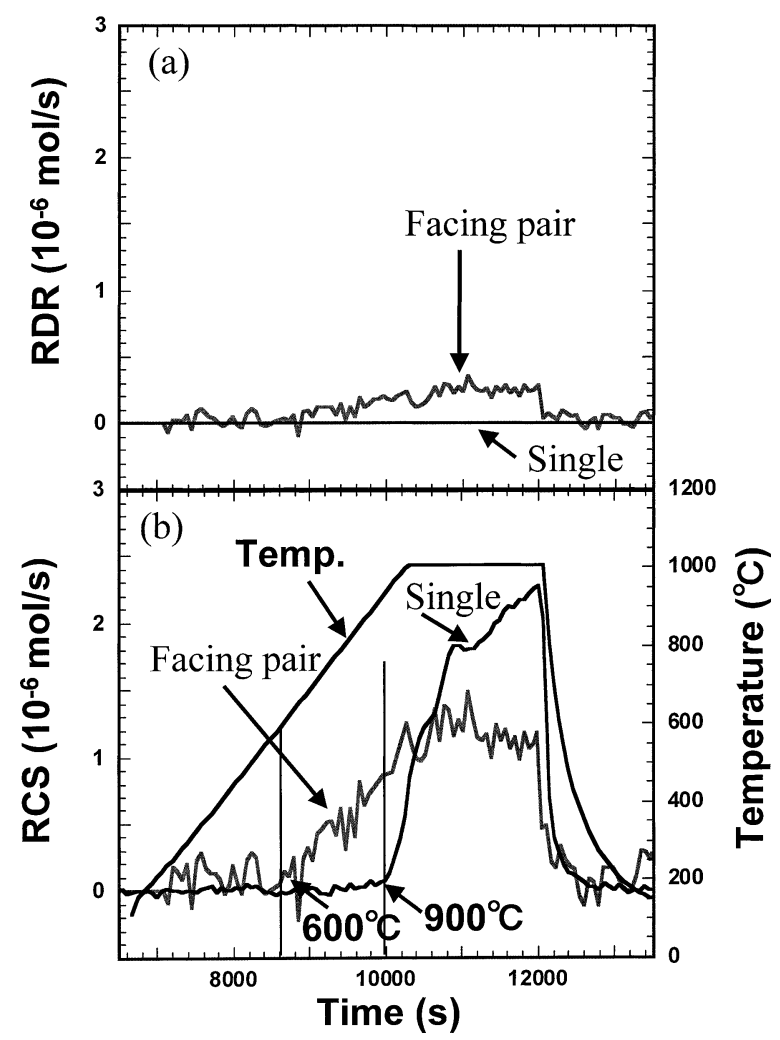

Fig. 12. Comparison of reaction rate between the facing pair and the single graphite in $\mathrm{Ar}-30 \mathrm{vol} \% \mathrm{CO}_{2}$.

the space between the two disks. The alignment of two disks affected the reaction rate significantly, so that the absolute value could not compared between two experiments in the different distance. In the case of the single graphite experiment, the alumina disk was more or less smaller and

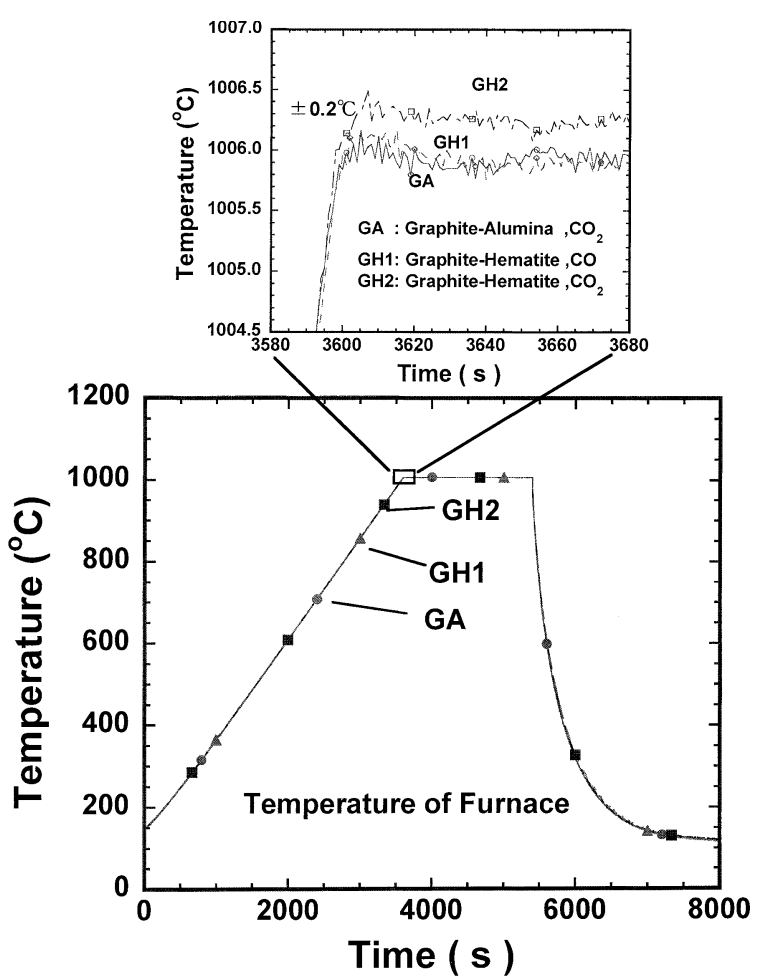

Fig. 13. Comparison of furnace temperatures among three experiments.

GA: Graphite-Alumina, $\mathrm{CO}_{2}$

GH1: Graphite-Hematite, $\mathrm{CO}$

$\mathrm{GH} 2$ : Graphite-Hematite, $\mathrm{CO}_{2}$

inclined. Then the gas flowing in the space between the graphite and the alumina disk might be larger than that of the facing pair. As a result, the maximum value of RCS in the single graphite was higher around $1000^{\circ} \mathrm{C}$. However in this study, the important thing is the starting temperature of RCS and it was $900^{\circ} \mathrm{C}$ in the single graphite, which was about $300^{\circ} \mathrm{C}$ higher than that in the facing pair. In addition, we have carried out the experiment with the narrower distance of $0.5 \mathrm{~mm}$. The tendency was the same between $1.2 \mathrm{~mm}$ and $0.5 \mathrm{~mm}$, however, the absolute value became smaller in the smaller distance, $0.5 \mathrm{~mm}$, because the amount of gas flowing into the space decreased.

\subsection{Temperature Change in the Reaction Surface by the Reaction Heat}

The temperature changes were measured during experiment to clarify the effect of reaction heat on the surface temperature where the reaction occurred. In Fig. 4, the positions of each thermocouple were illustrated.

Figure 13 shows the variations of the furnace temperature that were measured by B-type thermocouple in the alumina protection tube. In the lower part of Fig. 13, the temperatures in three kind of experiments (GA: GraphiteAlumina, $\mathrm{CO}_{2}$ gas, GH1: Graphite-Hematite, $\mathrm{CO}$ gas, GH2: Graphite-Hematite, $\mathrm{CO}_{2}$ gas) were plotted simultaneously. The three plots made one line in the lower figure of Fig. 13, which showed the quite accurate temperature control was attained. The upper figure in Fig. 13 magnified the small region around $1000^{\circ} \mathrm{C}$ and it was found that the difference of temperature among three experiments was $\pm 0.2^{\circ} \mathrm{C}$. From these results, the furnace temperature was used as the reference temperature and the temperature in 


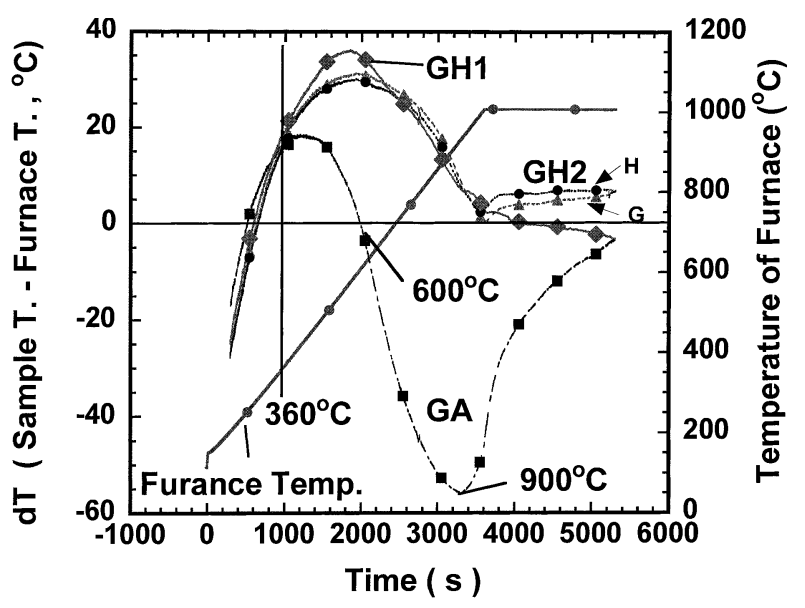

Fig. 14. Comparison of the temperature difference between sample surface and furnace temperature. The temperature of sample surface (Sample T) means that the graphite surface for experiments GA and GH1, and both of the surface of hematite and graphite for experiment GH2. In the case of GH1, the T.C. in the hematite surface was broken by the metallic iron formation.

the reaction surface measured by K-type thermocouple was compared each other.

Figure 14 shows the temperature variation of reaction surface, which was expressed as the temperature difference $(d T)$ from the furnace temperature $(d T=$ Sample T. Furnace T.). If there was no reaction, the difference would be zero or constant. The positive value corresponds to the exothermic reaction and the negative value means the endothermic reaction. Since the measurement error in the low temperature range by B-type thermocouple is relatively larger than that of K-type, the temperature difference under $360^{\circ} \mathrm{C}$ would not be so accurate. It could be understood from the result that all data under $360^{\circ} \mathrm{C}$ was in a line. The line expressed as ' $G A$ ' is a temperature difference of graphite in the Graphite-Alumina pair (the single graphite). The line shows negative value from about $600^{\circ} \mathrm{C}$, which means the endothermic reaction of gasification reaction occurred. Although the temperature $600^{\circ} \mathrm{C}$ might be relatively low temperature for gasification in single graphite, as the measurement error between K-type and B-type was not corrected, the temperature would not correspond the starting temperature of gasification exactly. On the other hand, the lines expressed as ' $\mathrm{GH} 1$ ' and ' $\mathrm{GH} 2$ ' are the temperature differences of hematite-graphite pairs in $\mathrm{CO}$ and $\mathrm{CO}_{2}$ flowrate, respectively. The both of temperatures in hematite and graphite pair were plotted in GH2 in Fig. 14, however, the single temperature of graphite surface in GH1 was plotted, because the thermocouple in the hematite was broken because of the formation of metallic iron. The values were always positive, which meant the exothermic reaction by reduction was dominant. However, it would be strange that the exothermic (reduction) and endothermic (gasification) reaction must occur simultaneously in separate position and the amount of heat of endothermic reaction should be larger than that of exothermic reaction (reduction). Then, the two temperatures at the hematite and the graphite surfaces in the facing pair should show a large difference. However, the result was different from the expectation and both the temperatures were varying with the same way keeping a small

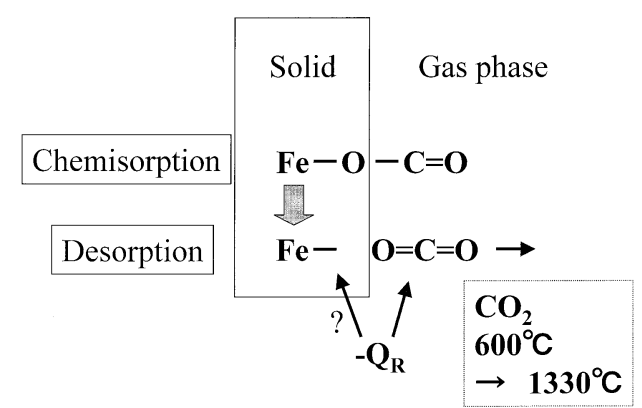

Fig. 15. A mechanism for the transfer of reaction heat. The problems are when the reaction heat generate and where the reaction heat is assigned.

discrepancy. The detail mechanism will be discussed in following paper. It can be concluded at this stage that the reason why the starting temperature of gasification decreased to $600^{\circ} \mathrm{C}$ in the hematite-graphite pair was that the reaction heat of reduction affected on the rate of gasification.

One possible mechanism which the temperatures of respective surfaces varied with same tendency could be explained by the way of heat transfer from opposite side.

The problems related to the reaction heat evolution would be as follows;

(1) when the reaction heat generates (at the chemisorption or at the desorption) and

(2) to where the reaction heat is assigned (solid side or gas phase).

Figure 15 is a illustration of a mechanism about the instance of reaction heat evolved. In general, most of reaction heat in solid-gas reaction was assigned on the solid side. However, if the reaction heat generates at the instance when the $\mathrm{CO}_{2}$ formed, it would be possible to assign to the gas phase $\left(\mathrm{CO}_{2}\right)$, and the temperature of $\mathrm{CO}_{2}$ gas would increased. For example, when the reaction temperature is $600^{\circ} \mathrm{C}$ and the whole heat of hematite reduction is assign to $\mathrm{CO}_{2}$ generated, the temperature of $\mathrm{CO}_{2}$ will be around $1300^{\circ} \mathrm{C}$ in maximum. If this mechanism is working on the hematite-graphite facing pair, the temperature of graphite surface, where the endothermic reaction (gasification) is occurring, can increase.

\section{Conclusion}

The simultaneous reaction between reduction of iron oxide and gasification of carbon was examined using hematite-graphite facing pair, which is to clarify the interaction of reactions occurring in the separated place. From the definition of coupling phenomenon, the discussion about the produced energy related to the production of entropy was carried out in the view point of two aspects that are (a) the gas composition change and (b) the evolution of reaction heat. The obtained results are as follows:

(1) Coupling phenomenon between reduction and gasification existed. The coupling reaction was the reduction reaction and the coupled reaction was the gasification reaction in $\mathrm{Ar}-30 \mathrm{vol} \% \mathrm{CO}$. On the other hand, it was opposite in $\mathrm{Ar}-30 \mathrm{vol} \% \mathrm{CO}_{2}$.

In $\mathrm{Ar}-30 \mathrm{vol} \% \mathrm{CO}$ :

(2) The starting temperature of reduction was $250^{\circ} \mathrm{C}$ in the hematite-graphite facing pair, while the temperature 
was $420^{\circ} \mathrm{C}$ in the single hematite.

(3) The gasification of graphite started from the same temperature $\left(250^{\circ} \mathrm{C}\right)$ as the reduction in the facing pair, while the gasification never occur without coupling phenomenon.

In $\mathrm{Ar}-30 \mathrm{vol} \% \mathrm{CO}_{2}$ :

(4) The starting temperature of reduction was $600^{\circ} \mathrm{C}$ in the hematite-graphite facing pair, while the reduction never occur without coupling phenomenon.

(5) The gasification occurred from $600^{\circ} \mathrm{C}$ in the facing pair, on the other hand, the starting temperature of gasification in the single graphite was $900^{\circ} \mathrm{C}$.

From the measurement of temperature in the reaction surface:

(6) The temperature of graphite surface increased in the case of the hematite-graphite facing pair. It could be explained that the starting temperature of gasification decreased in the facing pair according to the reaction heat from reduction.

\section{Acknowledgments}

The present study has been carried out in the research group of 'Project for the innovational ironmaking reaction in new blast furnace at half energy consumption and minimum environmental influences' supported by Special Coordination Founds of the Science and Technology Agency of the Japanese Government. The authors would like to express great appreciation to the member of research group for the valuable discussions.

\section{REFERENCES}

1) I. Prigogine and R. Defay: Thermodynamique Chimique, Liege, (1950). Japanese translation by Manabu Seno-o: Chemical Thermodynamics, Misuzu Shobo, Tokyo, (1968).

2) De Donder: Bull. Ac. Roy. Belg. Cl. Sc., 7 (1922), No. 5, 197.

3) W. J. Moor: Physical Chemistry 4th ed., translated into Japanese by R. Fujishiro, Tokyo Kagaku Dojin, Tokyo, (1978), 290.

4) M. Otani: Metallurgical Thermodynamics (Testu-Yakin Netsurikigaku in Japanese), Nikkan Kogyo Shinbun, Tokyo, (1973), 68, 96.

5) M. Chase: JANAF Thermochemical Tables, 3rd ed., Amer. Chem. Soc., Washington DC, (1986).

6) Y. Kashiwaya and K. Ishii: Tetsu-to-Hagané, 76 (1990), 1254

7) Y. Kashiwaya and K. Ishii: ISIJ Int., 31 (1991), 440.

8) Y. Kashiwaya and K. Ishii: Tetsu-to-Hagané, 79 (1993), 1305.

9) Y. Kashiwaya and K. Ishii: Tetsu-to-Hagané, 79 (1993), 1311. 